\title{
Local Networks and Regional Development: Features and Perspectives
}

\author{
Rosella Nicolini ${ }^{x}$ \\ IRES - Département des Sciences Economiques \\ Université catholique de Louvain
}

September 24, 2000

\begin{abstract}
A bstract
The aim of this paper is to draw attention to the role of local industrial networks in a regional development process. We build a oneregion model in which we investigate the conditions that make local networks arise, distinguishing between spontaneous and sustained networks. Accounting for the dixerence between incomplete and full coordinated agreements among ..rms, we establish the values of parameters that allow for the rise of each type of network. Finally, we argue on the positive exects that local networks yields to the regional internal product, making of them a reliable support for the regional economic take-ox.

K eywords: Industrial Networks, Local Weffare, Regional Development, Scale Economies.
\end{abstract}

J EL Classi..cation: E23, L16, R11, R13.

a thank Philippe Monfort and J acques F. Thisse for helpful comments and suggestions. This research is also a part of the Belgian Program on Interuniversity Pole of Attraction (PAI n.P4/01). 


\section{Introduction}

As it is often argued, the regional distribution of industrial activities inside the European Union (EU) is not uniform (see Amiti 1999). Indeed it displays large areas in which industrial clusters of ..rms concentrate. If one deeps inside the structure of the European clusters, two main structural forms appear. One type looks like a simple physical agglomeration of autonomous ..r.ms in bounded areas (eg. various diversi..ed cities as London, New York, Paris), while the other type appears as a form of coordination device among .r.rms. In the second case, clusters are endowed with various inter-..rm organizations. An example of this second kind of clusters are the industrial networks which include the Marshallian industrial districts.

We concentrate our analysis on this second kind of clusters since they display interesting features as local forms of aggl omerations. Indeed they seem to foster the economic development of the local environment in which they are settled. A few stylized facts help in supporting this statement. One of the most important case-studies is the economic reconversion of Wales (OECD 1996 and Cooke and Morgan (1998)). The Welsh Development Agency (the agency in charge of the reorganization of the industrial activity in Wales) accomplishes its tasks by sustaining thecreation of local networks of ..rms in order to make them the preferential suppliers of multinational subsidiaries. A similar decision has also been adopted by the European Union, when it needed to plan programs for the economic reconstruction in Kosovo and the Balkans (for instance, Obnova, Tafko). ${ }^{1}$ In those programs, it is recommended that the foreign investments should target the rise of a local industrial system, preferably as industrial networks, in order to be the engine of the local economic development. Furthermore, industrial networks keep on having a basic role in the economic systems of some of the most dynamic (and leading) European regions, for instance Baden Württemberg, Lombardia, Rhône Alpes.

This paper aims at developing a theoretical framework for these forms of organizations. In particular we intend to investigate (i) the conditions that make local networks arise either as spontaneous or sustai ned coordination devices among ..r.ms and (ii) the exects they may exert on the local environment.

By sustained networks, we mean the associations of .r.rms which are created and supported by local authorities, while the spontaneous ones are fre forms of agrement among ..rms. Sustained networks appear whenever the market conditions do not allow for the rise of spontaneous ones. Their start-up relies on the choices of a social planner who takes his decisions evaluating regional welfare ${ }^{2}$

Intuitively ..rms agree in belonging to spontaneous networks when they succeed in exploiting some externalities at group leve. Therefore, we assume that in this kind of

\footnotetext{
${ }^{1}$ See various references on the web, at the site http:// europa.eu.int/ com/ scr/ cgi/ framell.pl.

${ }^{2}$ Its interventions aim at improving the regional welfare by increasing the amount of good available for consumption.
} 
networks ..rms take advantage in coordinating their activities because of agglomeration forces stemming from a sharing knowledge process. A sharing knowledge process (that relies on a learning by doing mechanism) lets ..rms develop more rapi dly speci..c skills for the production process. Our model is built in such a way that a sharing knowledge process among ..r.ms takes place each time a network arises. Normally, this process involves the presence of an inter.r.rm organization that gathers the knowledge or experiences accumulated by each associated ..r.m and makes them available to the others, through the creation of exclusiveservices. For instance, a few years ago, in Northern I taly a group of ..rms close to Varese agreed in creating a University pole (LIUC), with the purpose to foster a more and more strictly collaboration between the local industrial and academic milieu.

As a consequence of this activity, for both types of networks, we assume that ..r.ms that join a network have to pay association fees for covering the costs of the services supplied by the network (for instance marketing or consulting services). The level of the associations fees combined with the amount of services granted will ensure the rise of the networks. ${ }^{3}$ In this perspective, these fees are introduced as excluding disclosure rules. Admitting excluding disclosure rules means that only the members of the association can bene.t from the advantages of the sharing information system, since they pay for them. As shown in Vives (1990), such fees-rules are needed for ensuring the stability of an agrement among ...rms. In our context, these fees prove to be a crucial feature that allows ..rms for switching from an independent status to an associated one and screening between spontaneous and sustained networks. In general, both spontaneous and sustained networks make the regional welfare increase, in comparison to a situation in which the same number of ..rms are autonomous. Moreover, through a numerical analysis, we con. r.rm that sustained networks may foster the local welfare even under the assumption of incomplete networks (i.e networks which do not include all the existing .r.ms). Indeed the role of a planner reveals to be as much crucial as its choices are in accordance with the preferences of the ..r.ms.

We develop this analysis by applying a monopolistic competition framework. This choice relies on some stylized facts. According to them, an agglomeration of ..r.ms (such as a local cluster) specializes in the production of a speci..c product, as in the case of Italian industrial districts. Nevertheless, each ..rm belonging to it develops technical skills in producing one of the possible varieties.

The remainder of the paper is organized as follows. In the next section we deal with the basic features of our model and Section 3 concentrates on the costs and bene.ts stemming from a network structure. Section 4 focuses on the conditions that allow for the rise of full coordinated spontaneous networks. Section 5 conveys the attention to the main features of sustained networks as well as the position undertaken by a central

\footnotetext{
${ }^{3}$ They represent a sort of sunk costs for the ..r.ms.
} 
social planner. Section 6 deals with the comparison between spontaneous and sustained networks when allowing for incomplete agreements among ..rms and Section 7 condudes.

\section{The model: basic features}

The main features of our model follow some of the principal building blocks of a general model in the economic geography literature. ${ }^{4}$ We consider a world in which there is one region. Our choice is driven by the interest to concentrate exclusively on some local determinants sustaining the creation of local networks. ${ }^{5}$ In the region, ...rms have to evaluate the opportunity either to compete autonomously on the market or to organize in industrial networks. At the beginning we assume that all of them take the same decision simultaneously and then we will relax this assumption.

In this region, all population is hired in local activities. There is full employment. The local population is thought as a continuum of consumers [0; $\mathrm{N}$ ], such that the level of consumption of each of them is negligible In the region, there is also a continuum of $n_{i} . . r m s{ }^{6}$ over the interval $[0 ; 1]$; each of them is negligible to the market. They produce a continuum of varieties of a single manufactured good. ${ }^{7}$ All of them behave as monopolistic competitors à la Dixit-Stiglitz (1977).

When ..rms behave independently on the market, all the population is hired in manufacturing activities. On the contrary, when an industrial network takes place, a service sector appears and a group of workers moves from the industrial sector to the service sector. ${ }^{8}$ This sector supplies a range of services to ..r.ms belonging to a network. For these services each ...rm has to pay some fees.

The utility function $\mathrm{C}(\mathrm{h})$ of each consumer is a CE S function and it is :

$$
C(h)={ }_{0}^{\cdot Z_{1}} \mathrm{C}(\mathrm{i})^{\frac{3 / 4}{3 / 4} 1 / 4} d i^{\frac{3 / 4}{3 / 4} 1} \text { with } 3 / 4>1:
$$

This function consists of a composite index which is generated by the combination of the consumption of dixerent varieties (of the samemanufacturing good). Each consumer displays a preference for variety and he consumes $c(i)$ unities of each of the $n$ varieties, according to an elasticity of substitution equal to $3 / 4>1$. When $3 / 4$ becomes arbitrarily

\footnotetext{
${ }^{4}$ See Fujita et alii. (1999) for references.

${ }^{5}$ In the. .nal conclusions we add some ideas on some possible extensions of this framework in a broader setting.

${ }^{6}$ Since we do not account for the free entry condition, we assume that there exists a . xed number (n) of ..rm in the region.

${ }^{7}$ As a consequence all unknowns are described by density functions as in Fujita et alii (1999).

${ }^{8}$ Wages are determined simultaneously allowing for migration across the sectors.
} 
large, the dimerentiated varieties are very closed substitutes, while for $3 / 4$ near to one varieties are poor substitutes.

We allow ..r.ms to make positive pro. .ts and we assume that consumers/ workers are also shareholders. In other words, consumers have a double source of income Indeed each of them supplies his unit of time in labor at wage $w$ and, at the same time, he receives a fraction of pro..ts (1/4i)) made by each ..r.m. So, the whole income of each consumer/ worker $(\mathrm{Y}(\mathrm{h}))$ is given by :

$$
Y(h)=w+Z_{0} \frac{1 /(4 i)}{N} d i:
$$

Each consumer devotes all his income to consumption :

$$
Y(h)={ }_{0}^{\cdot Z_{1}} p(i) c(i) d i
$$

where $c(i)$ is the level of consumption of each variety and $p(i)$ its corresponding price. Each consumer faces the following maximization problem:

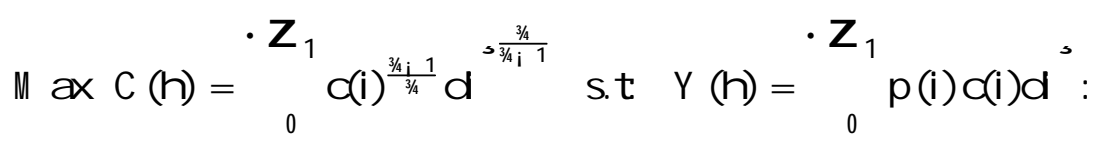

Using the FOCs, for any pair $(i ; j)$ of varieties we obtain:

$$
\begin{aligned}
& \cdot Z_{1} \mathrm{c}(\mathrm{i})^{\frac{3 / 41}{3 / 4}} \mathrm{di}{ }^{\left(\frac{3 / 4}{3 / 4} 1 \mathrm{i}\right)} \mathrm{c}(\mathrm{i})^{\left(\frac{3 / 4}{3 / 4} \mathrm{i} i 1\right)} \mathrm{i}, \mathrm{p}(\mathrm{i})=0 \text {; } \\
& \cdot Z_{1} c(i)^{\frac{3 / 4 / 4}{3 / 4}} d i^{\left(\frac{3 / 4}{3 / 4} i 1\right)} c(j)^{\left(\frac{3 / 4}{3 / 4} i 1\right)} i, p(j)=0 ;
\end{aligned}
$$

such that

$$
c(i)=\frac{p(j)}{p(i)}^{3 / 4} c(j):
$$

The.r.rst order conditions of this maximization problem give equality of the marginal rate of substitution to the price ratio. We de. ne the general price index $P$ as: 


$$
P=\int_{0}^{\cdot Z_{1}} p(i)^{1_{i} 3 / 4 d i} i^{\frac{1}{1_{i} i^{3 / 4}}}:
$$

The price index $\mathrm{P}$ corresponds to the minimum cost for purchasing a unit of the composite consumption good C(h) (Fujita \& allii (1999)). So, knowing that all the income is devoted to consumption, the total income $Y(h)$ is equivalent to the total expenditure and it may be rewritten as follows:

$$
Y(h)=P C(h)) \quad C(h)=\frac{Y(h)}{P}:
$$

By replacing (4) and (6) into (1), we obtain :

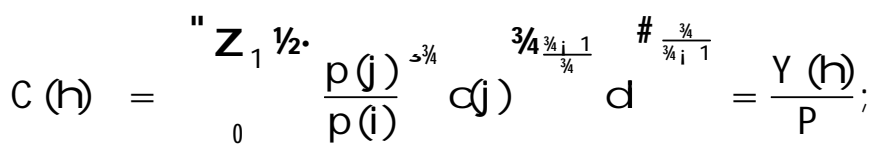

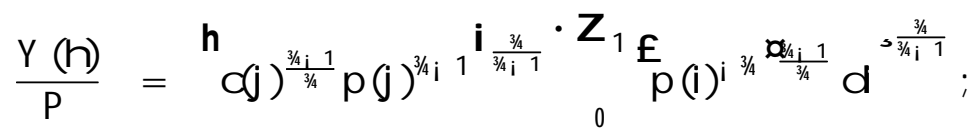

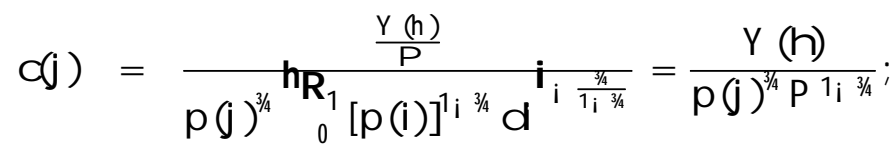

so that the demand function for the i i th variety of goods becomes:

$$
c(i)=\frac{p(i)}{P 1_{i}{ }^{3 / 4}}{ }^{i / 4} Y(h):
$$

Next, consider a particular ..rm producing a variety. Since all .r.rms are identical, each of them is expected to maximize the following pro.t. function $1 / 4 \mathrm{i}$ ); facing a given wage rate $\mathrm{w}$ :

$$
1 / 4 i)=p(i) x(i) i \quad k(i) \text {; }
$$

with

$$
x(i)=Z_{0}^{Z_{N}} c(i) d h=N c(i) \text {; }
$$


where $x(i)$ is the quantity produced by each .r.rm, $p(i)$ its corresponding price and $k(i)$ the cost function.

The technology adopted by a single ..r.m is:

$$
L(i)={ }^{-}+{ }^{\circ} x(i) \text {; }
$$

where $L(i)$ is the number of workers hired by a ..rm (i) to produce $x(i)$ units of variety (i) (with ${ }^{-}-$..xed costs- and ${ }^{\circ}$-marginal costs- positive), so that the cost function for a .r.rm (i) corresponds to:

$$
\mathrm{k}(\mathrm{i})=\mathrm{wL}(\mathrm{i})=\mathrm{w}\left({ }^{-}+{ }^{\circ} \mathrm{x}(\mathrm{i})\right):
$$

Plugging (9) and (10) into (8), we maximize the resulting pro..t function following Dixit-Stiglitz (1977). Each ..r.m chooses its price taking the price index $\mathrm{P}$ as given. Assuming nominal wages as numéraire $(\mathrm{i} . \mathrm{e} \mathrm{w}=1) ;{ }^{9}$ each ..r.m chooses its selling price at

$$
p(i)=\frac{3 / 4}{3 / 4 i} 1^{\text {q }} \text {; with } 3 / 4>1 \text { : }
$$

Given the demand function as (7) and applying (2), the market dearing condition for one variety (and by symmetry for all the others) may be written as:

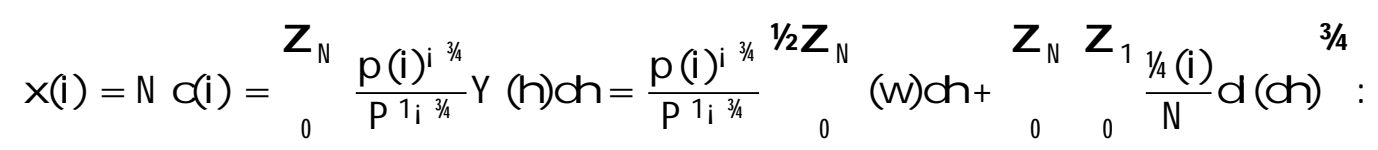

Therefore, via (5) and (11), we know that, at the equilibrium, the price index coincides with the price ..xed by a single .r.rm and equation (12) reduces to:

$$
x(i)=\frac{1}{p(i)}\left[N+p(i) x(i) i^{-} i^{\circ} x(i)\right] ;
$$

so that, after some al gebraic manipulations, the equilibrium output of each ..rm appears as :

$$
x(i)=\frac{N_{i}^{-}}{\circ},
$$

where we assume $\mathrm{N}>^{-}$:

\footnotetext{
${ }^{9}$ This assumption is allowed by the absence of unemployment and homogeneity of the workforce. Indeed, these assumptions feature a situation in which the labor market is a perfect competitive market.
} 
The numerator of equation (13) embodies a clear tradeox between the size of the market $(\mathrm{N})$ and the . xed costs of .r.rms $\left(^{-}\right)$, while the marginal costs of production (i.e. the denominator) exhibits negative exects. Finally, plugging equation (11) and (13) into (8), we are able to determine the level of pro..ts for each ..r.m at the equilibrium as:

$$
\begin{aligned}
1 / 4 \mathrm{i}) & =p(\mathrm{i}) \times(\mathrm{i}) \mathrm{j}(\mathrm{i})=p(\mathrm{i}) \times(\mathrm{i}) \mathrm{i}^{-} \mathrm{i}^{\circ} \times(\mathrm{i}) \\
& =\frac{3 / 4}{3 / 4 \mathrm{i} 1}\left(\mathrm{Ni}^{-}\right) \mathrm{i}^{-} \mathrm{i}^{-}\left(\mathrm{N}^{-}\right) ;
\end{aligned}
$$

which yieds

$$
1 / 4 \mathrm{i})=\frac{\left(\mathrm{N}_{\mathrm{i}}{ }^{-3 / 4}\right.}{(3 / 4 \mathrm{i} 1)} \text { with } \mathrm{N}>-3 / 4
$$

As expected, pro.ts are negatively axected by the degree of competitiveness $(3 / 4$ among ..rms.

\section{The costs and bene..ts of networks}

Wenow allow ..rms to createa network. A network consists in a more or less spontaneous agreement among. .rms by which they succeed in bene. .tting from positive externalities to exploit in their activity. Generally these externalities are connected with the exploitation of a sharing knowledge process. This process is often managed by an inter. .rm structure that is supposed to gather the information from the ac liates, in order to supply the most proper services. ${ }^{10}$ Indeed, for a ..r.m, joining a network means exchanging with the others its experience in production matters. A few studies con. r.rm that the spreading of information may follow diausion processes as those adopted in the innovation or marketing ..elds (see Mans. eld (1961) and de Palma et alii (1991)).

Since the sharing knowledge process represents an externality, we assume it exhibits its positive exects in reducing both the .xed and marginal costs of production in the spirit of Chipman (1970). Speci..cally, we assume that belonging to a network involves a sharing process that entails the use of particular services. Concretely, these services succeed in reducing the production costs by changing the technology. This exect is obtained by introducing $\tilde{A} 2[0 ; 1]$ as a parameter that arects the size of the technological coec cients of (9), when allowing for networks. ${ }^{11}$ The new technology function such that:

\footnotetext{
${ }^{10}$ In this sense, in this framework, the rise of network coincides with the start up of a service sector, whose interets are addressed to the following ..elds: marketing, consulting and training of the workforce.

${ }^{11}$ This new parameter can be also seen as the proportion by which to share the amount of cost savings generated by the creation of the network.
} 


$$
L^{N}(i)={ }^{-0}+\circ Q(i) \text {; }
$$

where

$$
{ }^{-0}=(1 ; \tilde{A})^{-}<^{-}
$$

and

$$
{ }^{\circ 0}=\tilde{\mathrm{A}}^{\circ}<{ }^{\circ} ;
$$

with Ã 2 [0; 1]; and, therefore, (15) will replace (9) each time a network appears in our region.

Moreover, we need to plan a device for granting services exclusively to the associated ..rms and make .r.rms pay for them. Following Vives (1990), one of the possible criteria is to introduce positive association fees (, ); with, $>0$. In the real world, when ..r.ms decide to form a network, the quotas they pay (for belonging to it) are normally devoted to ...nance the common activities of the network itself. As described in various reports (OECD (1996), Cooks-Morgan (1998)), the activities of the networks spread around three main directions: training of the labor force, consulting, marketing and export facilities. All these kinds of interventions aim at fostering the productivity (and competitiveness) of the network's ..rms in comparison to that of their competitors. Relying on these stylized facts, we will specify a cost function that accounts for both consulting and marketing facilities. In this case, we take into account the services supplied for strengthening the learning by doing process. In that sense, the fees paid by .r.rms allow for the organization of particular services in bureaucratic or marketing matters. In the spirit of this model, introducing these activities means creating a service sector in which employed people bene .t of the same treatment as manufacturing workers. Since the only factor of production of this model is labor, it is reasonable to consider that the costs of participation to a network structure ( , ) correspond to the total sum paid by all the ..rms to ..nance a service sector which engages workers at wage w: We assume that the cost of production, for ...rms belonging to a network, results from the linear combination between two forms of imposition. According to the magnitude of a parameter $\mu 2[0 ; 1]$, the association fees switch from the per-quantity level of imposition (for, $=0$ ) to the lump-sum one (for , =1). In particular, the cost function for network members transforms as follows (recalling that $\mathrm{w}=1$ )

$$
\begin{aligned}
\mathrm{k}^{\mathrm{N}}(\mathrm{i}) & =\mathrm{L}^{\mathrm{N}}(\mathrm{i})+\mu_{,}+(1 ; \mu), \mathrm{x}(\mathrm{i})={ }^{-0}+{ }^{\circ} \mathrm{g}(\mathrm{i})+\mu_{,}+(1 ; \mu), x(\mathrm{i}) \\
& =(1 ; \tilde{A})^{-}+\tilde{A}^{\circ} \mathrm{x}(\mathrm{i})+\mu_{,}+(1 ; \mu), x(i):
\end{aligned}
$$


So far, we de .ne the pro.tt function (1/4 (i)) for a network ..rm as:

$$
\begin{aligned}
{ }_{1 / 4}^{N}(i) & =p^{N} x^{N}(i) ; k^{N}(i) \\
& =p^{N} x^{N}(i) ; \tilde{A}^{0} x^{N}(i) ;(1 ; \mu)(,) x^{N}(i) ;(1 ; \tilde{A})^{-} ; \mu,
\end{aligned}
$$

As before, by the maximization of pro.ts at .r.m level (as for equation (11)), the price (at the equilibrium) for any ..rm becomes:

$$
p^{N}(i)=\frac{\mu / 4}{3 / 4 i 1}\left[\tilde{A}^{0}+\left(1_{i} \mu\right)_{J}\right]:
$$

If we assume that all the .r.rms take the same decision simultaneously (i.e. full coordination), at the equilibrium, all prices will be identical and $s 0 p^{N}(i)=P^{N}$ :

Under the hypothesis of full coordination among ..rms, exploiting the market clearing condition (12) and plugging (18) into (12), we determine $x^{\mathrm{N}}$ (i) as the quantity produced by a .r.m (i) in a network at the equilibrium:

$$
x^{N}(i)=\frac{1}{p^{N}(i)} \stackrel{f}{N}+p^{N}(i) x^{N}(i) ;(1 ; \tilde{A})^{-} \text {i } \tilde{A}^{\circ} x^{N}(i) \text { i } \mu, i(1 ; \mu), x^{N}(i)^{\alpha} \text {; }
$$

which yiedds

$$
x^{N}(i)=\frac{N_{i}\left(1_{i} \tilde{A}\right)^{-} i \mu_{s}}{\tilde{A}^{\circ}+(1 ; \mu)}:
$$

In addition to the parameters that axect the quantity produced at the equilibrium when ..rms are independent, this quantity is negatively axected by the presence of the association fees (. ). Nevertheless, comparing (13) and (20), even in absence of fees the level of production of each .r.r.m (at the equilibrium) inside a network is larger than that as independent because of the presence of positive externalities $(\tilde{A})$. The level of pro.ts for a ..rm in a network is obtained by replacing (17), (19) and (20) into (8) and it displays as:

$$
\begin{aligned}
& { }^{1 / N}(i)=\left(p^{N} x^{N}(i) ; \tilde{A}^{\circ} x^{N}(i) ;(1 ; \mu)(,) x^{N}(i) ;(1 ; \tilde{A})^{-} ; \mu_{J}\right) ; \\
& =\frac{\left(N_{i}\left(1_{i} \tilde{A}\right)^{-} i \mu_{\jmath}\right)}{(3 / 4 i 1)} i\left(1_{i} \tilde{A}\right)^{-} i \mu_{s} \text {; }
\end{aligned}
$$

so that for any , 0 , 


$$
{ }_{1 / 4}^{N}(i)=\frac{\left.N i^{3 / 4}(1 ; \tilde{A})^{-}+\mu_{\lrcorner}\right]}{(3 / 4 i 1)}:
$$

This last equation looks very similar to (14). Inside a network, the pro. .ts of a ..rm are positively axected by the externalities issued from the agreement (namely $(1 ; \tilde{A})<1$ ) and negatively by the ..xed amount of fees $\left(\mu_{s}\right)$..r.ms have to pay for.

\section{The rise of spontaneous industrial networks}

In the previous section, we presented the basic features we chose for modelling a network structure as a general form of local agglomeration. Consequently, in this section, we point the attention to the conditions that make .r.ms freely decide to create industrial networks. As al ready discussed, we intend to disentangle between spontaneous and sustained industrial networks. The former class includes all networks that arise spontaneously as agreements among ..r.ms, while the latter corresponds to the networks that public authorities patronize with the purpose of fostering local development. One critical point that emerges from this distinction is to look at the conditions under which each of two categories take place. To this end, we intend to compare them in order to check if (i) they are self-excluding and (ii) the magnitude of their exects at a macro level is strongly dixerent.

A ssumption 1: Firms agree in creating a network each time it makes their pro..ts rise.

Firms create spontaneous networks if the pro.ts that networks carry out are higher than those which . r.rms reach independently. In this . r.st part, we keep on the hypothesis of full coordination in decisions among ..r.ms, while in another section we will relax this assumption.

The decision problem faced by ...rms leads to concentrate on the level of association fees $(\mathrm{s}$, ): the willingness to pay for participating to a network needs to be compensated by the bene.ts $(\tilde{A})$ they may receive

Firms . .x the critical level of fees for which they take advantage in joining a network by comparing the level of pro.ts ((14) and (21)) they may reach in the two scenarios. Indeed, the critical level of association fees is ..xed by solving the following inequality:

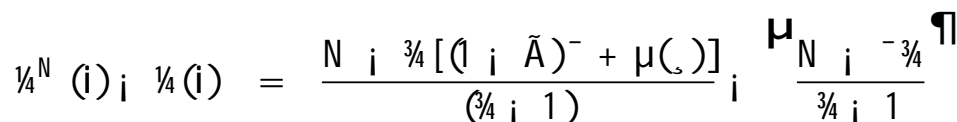

$$
\begin{aligned}
& =\frac{\left.3 / 4 \tilde{A}^{-} \mathrm{i} \mu(,)\right]}{(3 / 4 \mathrm{i} 1)}, 0, \mathrm{~F}^{\prime}, \frac{\tilde{\mathrm{A}}^{-}}{\mu} \text { : }
\end{aligned}
$$


P roposition 1 Independent ...rms agree in creating an industrial network whenever the level of association fees $($,$) satis. es the following condition: , ' , F{ }^{\prime}\left(\tilde{A}^{-}\right) \neq 1$ :

This condition means that the more ..rms have to pay association fees as ..xed costs (the higher $\mu$ is), the more pro.ts will be negatively axected by them and ..rms are less likely to create full coordinated spontaneous networks. The higher are the bene.ts driven by networks via a reduction of ..xed costs (see the magnitude of $\tilde{A}^{-}$; generated by the dixerence between ${ }^{-0}$ and ${ }^{-}$), the more .r.rms are likely to join full coordinated spontaneous networks. Firms are likely to join a network each time they can bene .t from a reduction of . .xed costs, merely paying per-quantity association fees. Indeed, for $\mu=0$, ,F ! 1 and .r.rms agree in creating a network for any value of the other parameters. Finally, it is useful to notice that when full coordinated networks appear, the degree of competition among .r.rms does not axect the decision dhoices.

\section{The role of a local social planner and the rise of sustained networks}

The creation of spontaneous networks relies on the decisions that ..rms take independently, just accounting for the fact that all of them participate in this association. On the contrary, it happens also that industrial networks arise by the direct intervention of a local authority, as discussed in the .r.st section. This authority decides how to create and organize them, according to its own evaluation of the economic welfare (RW) of the region as a whole This is the case of the so called imposed or sustained networks. In this context, a social planner tries to solve problems of market failures (or inec ciencies). We assume that the objective function of a social planner should take into account the total revenues of a region. Moreover, since a social planner cannot axect the level of prices in its region, its role involves to maximize the consumption function of the local population intended as:

$$
R W=Z_{0}^{Z_{1}} x(i) d i=\sum_{0}^{Z_{1} Z_{N}} c(i) d h d i
$$

Since all consumers are identical, the problem of the planner reduces to look at the utility (alias consumption) function of each consumer. When a planner thinks about the opportunity to make industrial networks arise, it compares the level of welfare with and without networks in order to ..x a critical level of association fees $(, \mathrm{p})$. Indeed the critical , $\mathrm{pi}$ value assesses the point at which the rise of a network does not produce any change in the whole regional production. Therefore, our aim is to distinguish the cases in which a planner may impose the rise of a network that axects positively the regional 
welfare outside the range of opportunity allowed by ..r.ms. In other words, we intend to disentangle the cases for which local networks produce positive exects on the regional economy as a whole.

In order to ..x the critical , $\mathrm{p}$ i value; since all ..rms are identical and, at the equilibrium, they produce the same quantity of dixerent varieties, we need to compare (20) and (13), which leads to :

$$
\begin{aligned}
& x^{N}(i) \text { i } x(i)=\frac{N i(1 ; \tilde{A})^{-} i \mu_{s}}{\tilde{A}^{\circ}+(1 ; \mu)(,)} ; \frac{N_{i}^{-}}{\circ} ; \\
& =\frac{\left[N^{\circ} \text { i }(1 ; \tilde{A})^{-\circ} i^{\circ} \mu_{,} \text {i } N \tilde{A}^{\circ} \text { i }(1 ; \mu), N+{ }^{-} \tilde{A}^{\circ}+{ }^{-}(1 ; \mu),\right]}{{ }^{\circ}\left(\tilde{A}^{\circ}+(1 ; \mu)(,)\right)}:
\end{aligned}
$$

The regional welfare can be improved by the rise of a network when:

$$
x_{i}^{N}, x_{i} \Rightarrow, \cdot, p^{,} \frac{{ }^{\circ}\left[(1 ; \tilde{A})\left(N_{i}{ }^{-}\right)+{ }^{-} \tilde{A}\right]}{(1 ; \mu)\left(N_{i^{-}}\right)+{ }^{\circ} \mu}>0
$$

P roposition 2 According to the objective function of a regional social planner, a sustained network arises for , i values that satisfy the following inequality

$$
, ., p^{\prime} \frac{{ }^{\circ}\left[\left(1_{i} \tilde{A}\right)\left(N_{i}{ }^{-}\right)+{ }^{-} \tilde{A}\right]}{(1 ; \mu)\left(N_{i}{ }^{-}\right)+{ }^{\circ} \mu} \text { : }
$$

As in the case of , $\mathrm{F}$, the condition (23) joins together the costs and bene .ts generated by a network, but in spite of the other case, in (23) they cannot be easily separated.

Comparing (23) and (22), it appears to be possible that the range of , $\mathrm{i}$ values for which sustained networks may arise is larger than that for spontaneous networks. This implies that a social planner has room to intervene in economic matters for bettering the total welfare of the region, under some conditions at which all the other market agents do not succeed in self-organizing in networks.

If we want to look more in detail at the conditions that can make, $\mathrm{p}>, \mathrm{F}$; we need to compare the correspondent expressions in order to obtain that:

$$
, p>, F) \frac{{ }^{\circ}\left[\left(1_{i} \tilde{A}\right)\left(N_{i}^{-}\right)+{ }^{-} \tilde{A}\right]}{{ }^{o} \mu+\left(1_{i} \mu\right)\left(N_{i}{ }^{-}\right)}, \frac{\tilde{A}^{-}}{\mu}
$$

or better

$$
, p>, F) \text { if }\left[\mu^{\circ}(1 ; \tilde{A}) i^{-} \tilde{A}(1 ; \mu)\right], 0 ; i: e: \tilde{A}<\frac{\mu^{\circ}}{\mu^{\circ}+{ }^{-}(1 ; \mu)}
$$


The relationship between the magnitude of,$p$ and,$F$ is mainly related to the tradeox between the bene.ts issued from a network (embodied in parameter Ã) and the costs it carries out (via parameter $\mu$ ). The social planner has room to intervene in economic matters of the region each timethe market conditions prevent ..r.ms from fredy associate. As a consequence of the relation detected above (24), a planner may impose the rise of a network when the creation of a network implies to sustain principally ..xed association fees in order to bene.t from the reduction of marginal costs of production (that is the meaning embodied in $\mu^{\circ}(1 ; \tilde{A})$ ).

\section{The case of incomplete networks}

In the previous section we dealt with the assumption of full coordination among .r.rms. That hypothesis involves the condition that all ..rms simultaneously decide to join a network. On the contrary, in this section, we investigatehow a network may rise drawing away that hypothesis. We assumethat only a proportion » (with » $2(0 ; 1)$ ) of all existing .r.rms decide to create a network. ${ }^{12}$ This new hypothesis allows for the simultaneous existence of independent and network ..rms, entailing a distinction in prices between the varieties produced by each group of .r.rms. Since .r.rms still choose their selling price as a mark-up over costs, the price index $P$ (hence labelled as $\bar{P}$ ) becomes:

$$
\bar{P}={ }_{0}^{\cdot Z} p^{N}(i)^{1_{i} 3 / 2 d i+}{ }_{\gg}^{Z_{1}} p(i)^{1_{i} 3 / 2 d i}{ }^{\frac{1}{1 i^{3} / 4}}:
$$

By equations (11) and (19), we state that all independent ..rms choose the same price $(p(i))$ and all the network ones $\left(p^{N}(i)\right)$, the price index $\bar{P}$ reduces to :

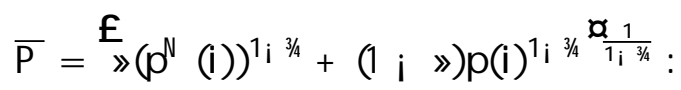

Consumers bene.t from a change in their disposable income due to the creation of the network. As a consequence, their demand function needs to be computed again to account both their new level of income and the variety diversi..cation. ${ }^{13}$ In particular, de. ning $\mathrm{x}(\mathrm{i})$ and $\mathrm{x}^{\mathrm{N}}$ (i) as (respectively) the demand addressed to an independent and a network ..rm, at the equilibrium, we determine them by solving the following system of equations (still accounting for $w=1$ ):

\footnotetext{
${ }^{12}$ When $\gg=0$ all ..rms are independent on the market, while for $\gg=1$ all ..rms belong to a full coordinated network.

${ }^{13} \mathrm{Namely}$, varieties produces by independent ..rms and those produces by .r.ms in network for which they pay dixerent prices.
} 


$$
\begin{aligned}
& \left.\sum^{8} x(i)=\frac{p(i) i^{3 / 4}}{(\bar{P})^{\left(1 i^{3 / 4} / 4\right.}} \stackrel{f}{N}+\left(1_{i} \gg\right)[1 / 4 i)\right]+»^{f} / / 4(i) \\
& >x^{N}(i)=\frac{p^{N}(i)^{i^{3 / 4}} f}{(\bar{P})^{\left(1 i^{3 / 4}\right.}} N+\left(1_{i} \gg[1 / 4 i)\right]+»^{f} \mathbb{H}_{4}^{N}(i)^{100}
\end{aligned}
$$

where:

$\left.{ }^{t} / 4 i\right)^{\prime}\left[\left(p(i) i^{\circ}\right) \bar{x}(i) i^{-}\right]$;

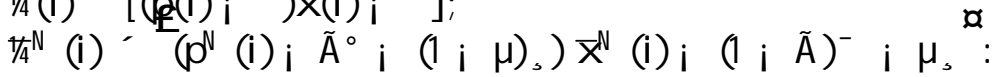

Each equation of this system corresponds to an application of the market clearing condition (12). Replacing (25) in the system and after some algebraic manipulations, the equilibrium conditions reduce to :

$$
\begin{gathered}
x(i)=B\left(A^{i^{1}}\right) \\
x^{N}(i)=D\left(C^{i}\right)
\end{gathered}
$$

$$
\begin{aligned}
& \text { where: }
\end{aligned}
$$

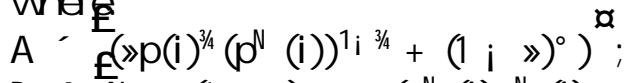

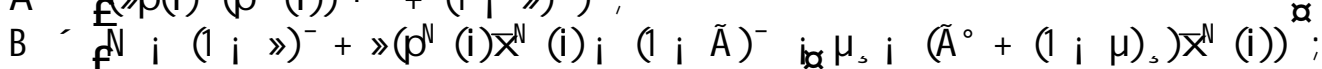

$$
\begin{aligned}
& C^{\prime}\left(1_{i} \gg\right) p(i)^{1} i^{3 / 4}\left(p^{N}(i)\right)^{3 / 4}+\gg\left(\tilde{A}^{\circ}+(1 ; \mu),\right)^{2} \text {; } \\
& D^{\prime}\left[N \text { i } \gg(1 ; \quad \tilde{A})^{-} i ~ \gg \mu_{\nu}+(1 ; \gg)\left(p(i) x(i) i^{-} i^{\circ} x(i)\right)\right]:
\end{aligned}
$$

Plugging (11) and (19) into the previous system, and knowing that it is a linear system in $\bar{x}(i)$ and $\bar{x}^{N}(i)$; we obtain the equilibrium demands. We label them as $\bar{x}(i)^{x}$ and $x^{N}(i)^{x}$ : Given the complex forms of these two expressions, we keep on indicating them with their symbols $\left(x(i)^{\alpha}\right.$ and $\left.x^{N}(i)^{x}\right)$ while in the Appendix we report the full algebraic expressions.

Once accounted for the equilibrium demand of each group of ..rms, we are able to determine the pro.ts they make The pro.ts $\left(\mathbb{T}_{4}^{N}(i)\right)$ made by one of the $\gg$ i firms belonging to the network are (via (19)) :

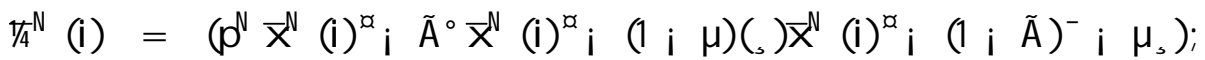

$$
\begin{aligned}
& =\frac{\left(\tilde{A}^{\circ}+(1 ; \mu),\right)^{N}}{(3 / 4 i 1)} x^{N}(i)^{\infty} \text { i }(1 ; \tilde{A})^{-} \text {i } \mu_{s} \text {; }
\end{aligned}
$$

and those ( $1 / 4 \mathrm{i})$ ) for any independent ..rm are (accounting for (11) :

$$
\begin{aligned}
\left.{ }^{1 / 4 i}\right) & =p(i) x(i)_{\uparrow}^{\infty} i^{-} i^{\circ} x(i)^{\infty} \\
& =\frac{\circ}{3 / 4 i} 11(i)^{\infty} i^{-}:
\end{aligned}
$$

The lump-sum association fees $\left(\mu_{\lrcorner}\right)$are a burden for the pro.ts of network ..rms. They exhibit a negative exect which should be compensated by some positive externalities $\left(\tilde{A}^{-}\right)$carried out by the network. As usual, a .r.rm decides to join a network by 
comparing the pro..ts it could make joining it or still being independent. In this case, it takes its decision by comparing (26) and (27). This criterion yields to the selection of a critical value ${ }^{-} \mathrm{F}$ for which ..r.ms agree in joining a spontaneous but incomplete network. Speci..cally, our objective is to look for the following condition:

$$
z_{4}^{N}(i), t_{4} \text { i) for, },-F \text { : }
$$

Since both these two equations are trascendental in , ; we cannot but ..nding a numerical approximation for ${ }^{-} \mathrm{F}$. By dealing with numerical simulations, ${ }^{14}$ we account for a few alternatives as key-parameters change. The table below contains the grid of results we obtained. We concentrate on how the critical values of fees vary both as lump sum or per-quantity fees and on the role of externalities carried out by networks (i.e whether they arect the ..xed or the marginal costs). We focus on four alternative cases. Case 1 and Case 2 deal with the presence of per quantity association fees. Yet, Case 1 principally deals with externalities involving a reduction of ..xed costs, while Case 2 those axecting the marginal costs. Conversely Case 3 and Case 4 concentrate on lump sum fees, still in presence of positive externalities on . xed costs (in Case 3) and on marginal costs (in Case 4).

Table 4.1: Critical values for ${ }_{, \mathrm{F}} 15$

\begin{tabular}{|l|l|l|l|l|}
\cline { 2 - 5 } \multicolumn{1}{c|}{} & Case 1 & Case 2 & Case 3 & Case 4 \\
\cline { 2 - 5 } \multicolumn{1}{c|}{} & $\mu=0: 2 ;$ & $\mu=0: 2 ;$ & $\mu=0: 8 ;$ & $\mu=0: 8 ;$ \\
\cline { 1 - 4 }$=0.1$ & 0.905231 & 0.373298 & 3.05715 & 1.1909 \\
0.2 & 0.904945 & 0.368673 & 3.04328 & 1.18963 \\
0.3 & 0.904665 & 0.364379 & 3.02867 & 1.18636 \\
0.4 & 0.90439 & 0.360382 & 3.01329 & 1.18414 \\
0.5 & 0.90412 & 0.356651 & 2.9971 & 1.18195 \\
0.6 & 0.903855 & 0.353163 & 2,98005 & 1.1798 \\
0.7 & 0.903594 & 0.349893 & 2,96211 & 1.17769 \\
0.8 & 0.903338 & 0.346823 & 2,94323 & 1.17562 \\
0.9 & 0.903086 & 0.343935 & 2,92337 & 1.17359 \\
\hline
\end{tabular}

Thenumerical results included in the previous tableallow for drawing some comments on the relationship between the critical value of the association fee $(, \mathrm{F})$ and the size of a network (»). In all the four cases, there is a clear inverse relationship between, $\mathrm{F}$ and ». The larger the network is, the lower the level of ${ }^{-} \mathrm{F}$ is: In other words, the more..r.ms

\footnotetext{
${ }^{14}$ We compute all the numerical simulations by applying the software Mathematica.

${ }^{15}$ Assuming that ${ }^{-}=2 ; \mathrm{N}=20 ; 3 / 4=4 ;{ }^{\circ}=1$ : Any change of the value of these parameters merely produces a size-exect on the resulting outcomes for , :
} 
decide to join a network, the smaller is the range of , $\mathrm{i}$ values for which they agree in

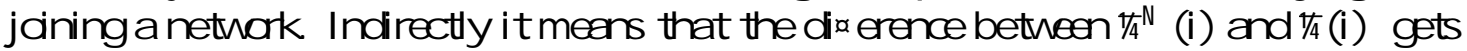
lower and lower as » increases. Admitting the presence of partial coordinated networks means allowing for a dixerentiation in the technology functions among ..rms. When the size of a network (») is very small, the network does not exert any substantial impact on the market outcomes. Therefore, pro. ts and production generated by independent ..r.ms remain nearly unchanged, while network .r.rms bene.t from their new technology (17) that makes their pro.ts increase. In that sense, network ..rms ... $x^{-}, \mathrm{F}$ as the value which cancels out the dixerence between their pro.ts and those of the other ..rms. As the size of the network (») increases, all the market agents become aware of the presence of the network and they react. So, the market outcomes adapt accordingly. These reactions erode the dixerential of pro. ts between network and independent .r.rms as well as the level of fees .r.rms are willingly to pay for belonging to a network. Indirectly, this relation implies that ..rms may take more advantages in joining small networks since they are likely to pay higher association fees.

As usual, the willingness to join a network relies on the tradeox between the advantages carried out by it and the burden of the association fees. In general, , ${ }_{F}{ }^{16}$ is greater for networks that allow for lump sum association fees (namely, the higher $\mu$ is). Moreover, the range of , $i$ values for which .r.rms may pro. tably join a network increases when networks succeed in reducing the ..xed costs of production (i.e the lower Ã is).

Following the same approach we proposed above for the full coordinated networks, we are determining the conditions that let spontaneous networks rise with those of sustained ones. As before, an hypothetical central planner looks at evolution of the regional welfare for eventually imposing the creation of a network. A planner simply cares about the utility of local consumers, since it cannot manage prices. When considering for the simultaneous presence of independent and networks ..r.ms, the regional welfare $\overline{\mathrm{RW}}$ transforms as follows:

$$
\overline{\mathrm{RW}}={ }_{0}^{\mathrm{Z}} \mathrm{x}^{\mathrm{N}}(\mathrm{i}) \mathrm{di}+{ }_{\gg}^{\mathrm{Z}_{1}} \mathrm{x}(\mathrm{i}) \mathrm{di}:
$$

At the equilibrium, we know that the level of production of each ..rm inside each group is identical, so $\overline{R W}$ reduces:

$$
\overline{\mathrm{RW}}=(\gg) \mathrm{X}^{\mathrm{N}}(\mathrm{i})^{\alpha}+\left(1_{\mathrm{i}} \gg\right) \mathrm{x}(\mathrm{i})^{\alpha}:
$$

In order to .x the critical level of association fees for partial coordinated networks, a planner needs to compare the regional welfare $\overline{\mathrm{RW}}$ with that achieved in presence of all independent ..rms. Its problem consists in . xing a critical value of ${ }_{,}^{-} p$ such that:

\footnotetext{
${ }^{16}$ So, the wider is the range of , $\mathrm{i}$ values for which ..rms take advantages in joining a network.
} 


$$
\left.\overline{R W}, \frac{N_{i}^{-}}{\circ}\right) \gg X^{N}(i)^{\infty}+(1 ; \gg) x(i)^{\infty}, \frac{N_{i}^{-}}{\circ} ; \text { for , . }, p_{s} \text { : }
$$

Because of the presence of trascendental equations in , , we concentrate on some numerical simulations. The criteria we follow are identical to those applied in the case of spontaneous networks. The following table contains the outcomes of these simulations.

Table 4.2: Critical values for ${ }^{-} \mathrm{p}^{17}$

\begin{tabular}{|l|l|l|l|l|}
\cline { 2 - 5 } \multicolumn{1}{c|}{} & Case 1 & Case 2 & Case 3 & Case 4 \\
\cline { 2 - 5 } \multicolumn{1}{c|}{} & $\mu=0: 2 ;$ & $\mu=0: 2 ;$ & $\mu=0: 8 ;$ & $\mu=0: 8 ;$ \\
\cline { 1 - 4 } & $\tilde{A}=0: 3$ & $\tilde{A}=0: 8$ & $\tilde{A}=0: 3$ & $\tilde{A}=0: 8$ \\
\hline 0.1 & 0.906841 & 0.414102 & 3.10758 & 1.20445 \\
0.2 & 0.906512 & 0.406244 & 3.09739 & 1.2017 \\
0.3 & 0.90619 & 0.396996 & 3.08679 & 1.19905 \\
0.4 & 0.905874 & 0.388978 & 3.07576 & 1.19643 \\
0.5 & 0.905565 & 0.381933 & 3.06429 & 1.19386 \\
0.6 & 0.905262 & 0.375673 & 3.05238 & 1.19135 \\
0.7 & 0.904965 & 0.370061 & 3.04 & 1,18889 \\
0.8 & 0.904674 & 0.36499 & 3.02715 & 1.18648 \\
0.9 & 0.904389 & 0.36038 & 3.01382 & 1,18413 \\
\hline
\end{tabular}

The numerical analysis of ${ }_{,}^{-} \mathrm{p}$ replicates the results we obtained for ${ }^{-} \mathrm{F}$ : The values of,$p$ reduces as the size of the network ( $\gg$ ) increases. The critical value ${ }_{,}^{-} p$ is chosen to keep -at least- unchanged the regional welfare when a network appears. The planner takes his decision knowing that the creation of a network carries out two levels of pro. ts, via a dixerentiation of the technology applied by ..r.ms. This condition allows for the existence of an economic surplus, stemming from the rise of pro.ts of network ..rms. Therefore, the fees it imposes allow for sharing across the market the surplus generated by network .r.rms. Being so strictly related to .r.rm pro..ts, , p evolves as , $F$ when the size of the network increases. Moreover, the planner recognizes how each group of ...r.ms axects mutually and how they contribute to the regional welfare. So far, it internalizes the transmission exect existing across ..r.ms and this allows it to ... ${ }^{-}, p$ greater than ${ }_{\lrcorner}, \mathrm{F}$ : In other words, even in the case of incomplete networks, a planner has room intervene in economic matters for improving the welfare of the region.

Even if both ${ }_{,}^{-} \mathrm{p}$ and ${ }_{,}^{-} \mathrm{F}$ reduces as the size of the network increases, it is interesting to detect how $\left(-, p_{-}, \mathrm{F}\right)$ varies with », or better how the room of intervention of the planner

\footnotetext{
${ }^{17}$ Assuming that ${ }^{-}=2 ; \mathrm{N}=20 ; 3 / 4=4 ;{ }^{\circ}=1$ : Any change of the value of these parameters merely produces a size exect on the resulting outcomes for, :
} 
changes. The most useful way to ..gure out this situation is to compare graphically the dixerence between the outcomes of the two previous tables, for each of the four scenarios. ${ }^{18}$

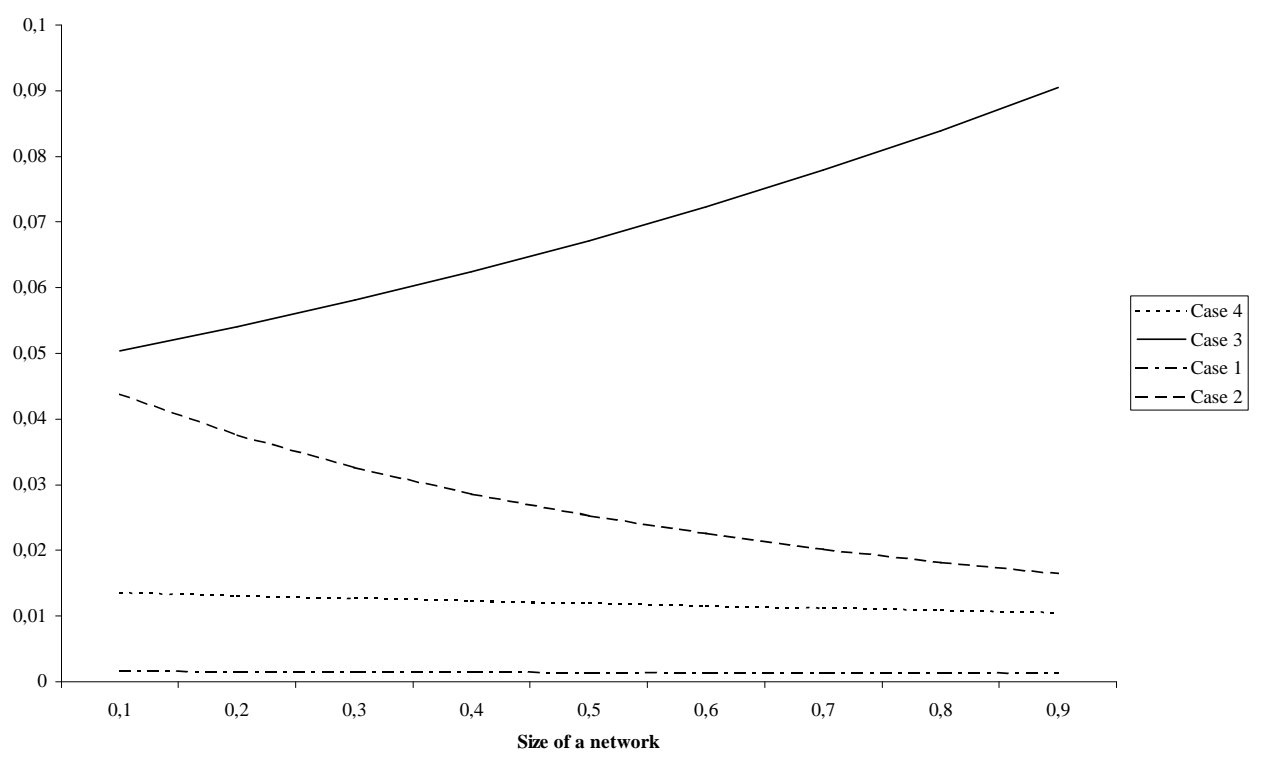

Figure 1: Dixerence between critical values of association fees and size of a network.

Looking at the previous ..gure, it is clear that the dixerence $\left(-, p-{ }_{-}, \mathrm{F}\right)$ is sensible to the size of the network just in two of the four cases, namely Case 2 and Case 3. In general, a planner succeeds in having more room for fostering the rise of a sustained network and, in particular a large network, each time its choices are in accordance with the preferences of ...rms. In other words, the dixerence $(-, p-, F)$ increases with $\gg$, when a planner proposes a network which mainly accounts for lump sum association fees and carries out consistent reductions of marginal costs of production. On the contrary, when allowing for networks that display per quantity association fees and involvea reduction of .xed costs of production, a planner may successfully foster just small sustained networks.

\footnotetext{
${ }^{18}$ It is important to remind that this comparison may suaer from some errors in numerical approximations (around $10^{\mathrm{i}}{ }^{6}$ ) due to the computation system of software packages.
} 


\section{Conclusions}

In this paper, we have tackled the problem of the creation of networks of ..rms in which the associated ..r.ms share a learning process. This process entails positive externalities that turn out to improve the technology of production for all the associated ..r.ms. Moreover, according to the magnitude of bene. ts entailed by networks, a full coordinated network will be preferred to an incomplete one. We distinguished between spontaneous and sustained networks and we derived the critical level of association fees that should be ..xed in order to make those networks arise. In the case of spontaneous networks, ..rms select the conditions underpinning the creation of networks by looking at their pro.ts. Conversely, sustained networks are usually created by local authorities with the target of fostering the local welfare Consumers take advantage of it since, in both cases, they get higher level of consumption, while pro. ts display positive variation only in the case of spontaneous network. However for both types of networks, the internal product of a region records positive variations. Under this viewpoint, all policies that support these organizations with the purpose to foster local development seem to be appropriate. Nevertheless, local authorities have to pay attention for the way in which to coordinate (or organize) these forms of activities according to their ...nal purpose Sustained networks succeed in helping the local development (as an increase in the internal product) under some conditions unacceptable to market agents. However, the bene.ts carried out by spontaneous networks are higher than those of sustained ones.

Among the possible extensions, it would be useful to develop some topics in policy matters, such as how to create a network, accounting for a wider international context. Allowing for other regions involves some changes in the structure of the model. The equival ence among workers-consumers-shareholders need to be rethought when admitting

free migration across regions. In addition, the demand addressed to each . r.rm should be tempered by the existence of other consumers and competitors whose role become crucial in accordance with the degree of integration of markets (see Fujita et alii. (1999)). In this enlarged setting, the opportunity to create a network should be reinforced whenever a network represents an opportunity to take advantage versus the other competitors. However, the mechanism that ..xes the level of association fees should account for other parameters, namely the degree of openness of the markets via the level of transport costs. Nevertheless, in a similar setting, it becomes crucial to detect how a network should improve (or sustain) the quota of production exported by each ..r.m.

\section{R eferences}

[1] M. A miti (1999): 'Specialisation Patterns in Europe', Weltwirtschaftliches Archiv, vol. 135 (4), pp.573-594. 
[2] J .S. Chipman (1970): 'External Economies of Scale and Competitive Equilibrium', Quarterly J ournal of Economics, vol. 84(3), pp.347-385.

[3] Ph. Cooke - K. M organ (1998): 'The Associational Economy', Oxford University Press.

[4] A. de Palma - J J. Droesbeke- C.Lefèvre (1991): 'Modèles de diausion en marketing' Paris, Presse Universitaire de France.

[5] A.K. Dixit and J . E. Stiglitz (1977): 'Monopolistic Competition and Optimum Product Diversity', American Economic Review, vol. 67, pp. 297-308.

[6] Fujita M .- K rugman P. - Venables A . (1999) 'The Spatial Economy', MIT Press.

[7] E.M ans..eld (1961): 'Technical Change and the Rate of Imitation', Econometrica, vol.29, pp.741-766.

[8] OECD (1996): 'Network of Enterprises and Local Development - Competing and Co-operating in Local Productive Systems', Local Economic and Employment Development Series

[9] X. V ives (1990): 'TradeAssociation disclosure rules, incentives to share information and weffare', Rand J ournal of Economics, vol. 21(3), pp.409-430.

\section{A ppendix}

In presence of partial coordinated networks, the system that allows to compute the equilibrium demands addressed to each ..rm of the two groups is the following:

$$
\begin{gathered}
x(i)=B\left(A^{i^{1}}\right) \\
x^{N}(i)=D\left(C^{i}\right)
\end{gathered}
$$

where

$$
\begin{aligned}
& A^{\prime},\left(f^{\prime} p(i)^{3 / 4}\left(p^{N}(i)\right)^{1} i^{3 / 4}+\left(1_{i} \gg\right)^{\circ}\right)^{\alpha} ;
\end{aligned}
$$

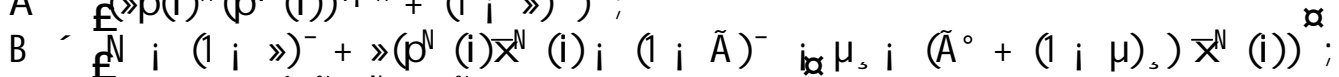

$$
\begin{aligned}
& C^{\prime}{ }^{1}\left(1_{i} \gg\right) p(i)^{1 / 3 / 4}\left(p^{N}(i)\right)^{3 / 4}+\gg\left(\tilde{A}^{\circ}+\left(1_{i} \mu\right)_{,}\right)^{2} \text {; } \\
& D^{\prime}\left[N \text { i } \gg(1 ; \tilde{A})^{-} i \gg \mu_{\nu}+(1 ; \gg)\left(p(i) x(i) i^{-} i^{\circ} x(i)\right)\right]:
\end{aligned}
$$

Solving this system (plugging also (11) and (19) into it)we obtain these results:

$$
\begin{aligned}
x(i)^{\infty} & =\frac{Q}{R} ; \\
x^{N}(i)^{\infty} & =\frac{M}{V} ;
\end{aligned}
$$


with

$$
\begin{aligned}
& Q=\frac{\left.h^{i^{3 / 4}\left[N_{i}\left(1_{i} \gg\right)^{-} i ~\right.} \gg\left(+, \mu+^{-}\left(1_{i} \tilde{A}\right)\right)\right]}{z_{i}\left(1_{i} \gg\right) h^{3 / 4}\left(h_{i}{ }^{\circ}\right)}+\gg h^{i^{3 / 4}}\left(k_{i} \quad\left(1_{i} \mu\right), i^{\circ} \tilde{A}\right)
\end{aligned}
$$

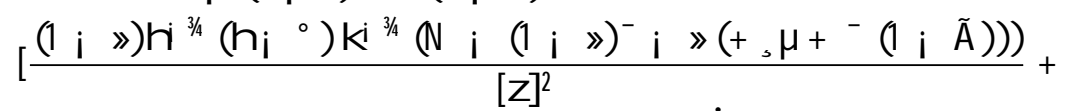

$$
\begin{aligned}
& \left.\frac{\mathrm{k}^{\mathrm{3} / 4}\left(\mathrm{~N}_{\mathrm{i}}\left(1_{\mathrm{i}} \gg\right)^{-} \mathrm{i} \gg\left(+, \mu+^{-}\left(1_{\mathrm{i}} \tilde{\mathrm{A}}\right)\right)\right)^{\mathrm{i}} 1_{\mathrm{i}} \mathrm{z}^{\mathrm{i}}{ }^{1}\left(1_{\mathrm{i}} \gg\right) \mathrm{h}^{\mathrm{i} / 4}\left(\mathrm{~h}_{\mathrm{i}}{ }^{\circ}\right)^{\Phi}}{\mathrm{z}}\right] \\
& R={ }^{i} z_{i}(1 ; \gg) h^{i / 4}\left(h_{i}{ }^{\circ}\right)^{\Phi} \\
& {\left[i \frac{(1 ; \gg) \gg h i^{3 / 4}\left(h_{i}{ }^{0}\right) k^{i^{3 / 4}}\left(k i \quad(1 ; \mu), i \tilde{A}^{\circ}\right)}{[z]^{2}}+\right.}
\end{aligned}
$$

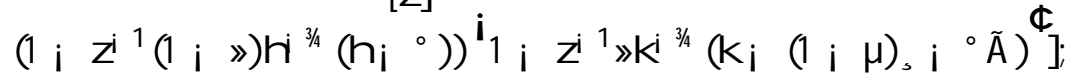

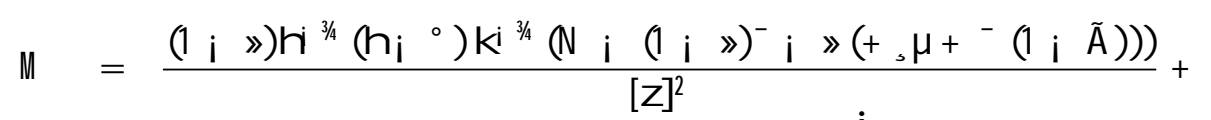

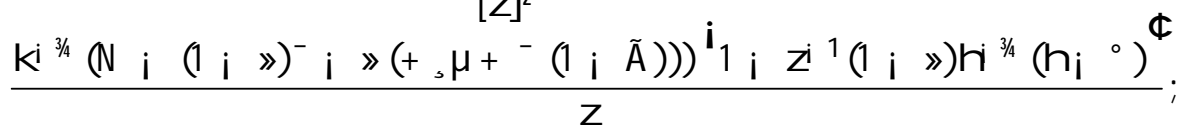

$$
\begin{aligned}
& V=\left[i \frac{\left.\left(1_{i} \gg\right) \gg h^{i^{3 / 4}\left(h_{i}{ }^{0}\right) k^{i^{3 / 4}}(k i \quad(1 ; \mu, ~} \tilde{A}^{\circ}\right)}{[z]^{2}}+\right. \\
& \left.\left(1_{i} z^{i}{ }^{1}\left(1_{i} \gg\right) h^{i^{3 / 4}\left(h_{i}{ }^{\circ}\right)}\right){ }^{i} 1_{i} z^{i} 1_{\gg k^{i}}{ }^{3 / 4}\left(k_{i} \quad(1 ; \mu), i^{\circ} \tilde{A}\right)^{\phi}\right]:
\end{aligned}
$$

knowing that

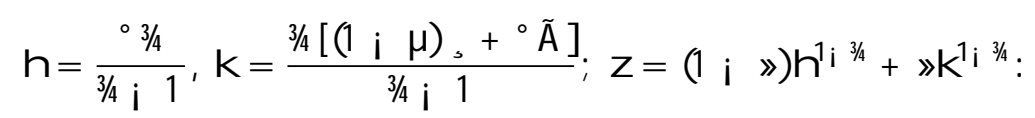

Comparing $\left(\mathrm{x}(\mathrm{i})^{\mathfrak{\alpha}}\right.$ and $\left.\mathrm{x}^{\mathrm{N}}(\mathrm{i})^{\mathrm{a}}\right)$, we establish that they are identical for :

$$
\left.\overline{\mathrm{x}(\mathrm{i})}^{\infty}={\overline{\mathrm{x}^{\mathrm{N}}(\mathrm{i})}}^{\infty}\right) \quad \gg=\frac{\mathrm{N}_{\mathrm{i}}^{-}}{-\tilde{\mathrm{A}} \mathrm{i}, \mu^{\prime}}
$$

and 


$$
\gg>0 \text { if } \mathrm{N}>- \text { and }, \frac{-\tilde{\mathrm{A}}}{\mu} \text { : }
$$

Moreover, when

$$
\left.»=0 \text { and } p^{N}(i)=p(i)\right) \quad x(i)^{\alpha}=x^{N}(i)^{\alpha}=\frac{N_{i}^{-}}{\circ} ;
$$

the equilibrium quantities coincide with those obtained in the case of all independent .r.ms and when

$$
\left.\gg=1 \text { and } p^{N}(i)=p(i)\right) \quad x(i)^{\alpha}=x^{N}(i)^{\alpha}=\frac{N i(1 ; \tilde{A})^{-} i \mu_{s}}{\tilde{A}^{\circ}+(1 ; \mu)}
$$

we ..nd the same results as in the case of a full coordinated network. 